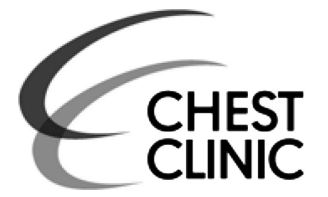

\section{(6) OPEN ACCESS}

'Department of Respiratory Medicine, Nottingham City Hospital, Nottingham, UK ${ }^{2}$ Southmead Hospital, North Bristol Lung Centre, Bristol, UK ${ }^{3}$ Department of Adult Critical Care, University Hospital of Wales, Cardiff, UK

${ }^{4}$ British Thoracic Society, London, UK

\section{Correspondence to} Dr W S Lim, Nottingham City Hospital, David Evans Building, Hucknall Road, Nottingham, Notts NG5 1PB, UK; weishen.lim@nuh.nhs.uk

This article is published simultaneously in BMJ Open Respiratory Research. Lim WS, Smith DL, Wise MP, et al. British Thoracic Society community acquired pneumonia guideline and the NICE pneumonia guideline: how they fit together. BMJ Open Resp Res 2015;2: e000091. doi:10.1136/ bmjresp-2015-000091

Received 2 February 2015 Revised 17 March 2015 Accepted 27 March 2015 Published Online First 14 May 2015

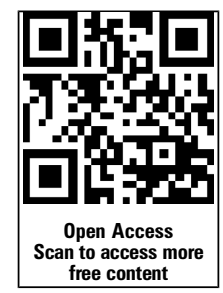
2015;70:698-700.

\title{
British Thoracic Society community acquired pneumonia guideline and the NICE pneumonia guideline: how they fit together
}

\author{
W S Lim, ${ }^{1}$ D L Smith, ${ }^{2}$ M P Wise, ${ }^{3}$ S A Welham, ${ }^{4}$ on behalf of the British \\ Thoracic Society
}

ABSTRACT

The British Thoracic Society (BTS) guideline for the management of adults with community acquired pneumonia (CAP) published in 2009 was compared with the 2014 National Institute for Health and Care Excellence (NICE) Pneumonia Guideline. Of the 36 BTS recommendations that overlapped with NICE recommendations, no major differences were found in 31 , including those covering key aspects of CAP management: timeliness of diagnosis and treatment, severity assessment and empirical antibiotic choice. Of the five BTS recommendations where major differences with NICE were identified, one related to antibiotic duration in low and moderate severity CAP, two to the timing of review of patients and two to legionella urinary antigen testing.

\section{BACKGROUND}

The British Thoracic Society (BTS) guideline for the management of adults with community acquired pneumonia (CAP) was published in $2009 .^{1}$ In December 2014, the National Institute for Health and Care Excellence (NICE) Pneumonia Guideline (CG191) was released. ${ }^{2}$ In this overview, the key differences between these guidelines are highlighted and plans for future guidance in this topic area are discussed.

\section{METHOD}

Following the release of the NICE Pneumonia Guideline, a small working group was convened under the auspices of the BTS Standards of Care Committee to review the BTS CAP Guideline recommendations taking into account NICE guidance. Each BTS recommendation was mapped to the relevant NICE Guideline recommendation(s), where applicable. Clinical judgement was applied to determine if any 'major differences' existed; in this context, the term 'major difference' is applied to denote a difference in the details of a recommendation that might mean a change in clinical practice, it does not describe the magnitude of any potential impact on clinical practice.

All BTS recommendations were annotated according to the following three levels: (1) Not included in NICE Guideline-BTS recommendation remains valid, (2) Overlap between NICE and BTS recommendations but no major difference existsBTS recommendation remains valid and (3) Overlap between NICE and BTS recommendations, some difference exists.

Due to differences in the methods used to grade the levels of evidence in each guideline, it was not possible to compare the strength of recommendations between guidelines.

\section{RESULTS}

The NICE Guideline contains 27 recommendations, of which 3 relate to hospital acquired pneumonia, one to lower respiratory tract infections including CAP and 23 specifically to CAP. The BTS Guideline contains 137 recommendations, all relating specifically to CAP. Of these 137 recommendations, 36 overlapped with NICE Guideline recommendations. Major differences with the NICE Guideline were considered to exist for only five BTS recommendations (see table 1).

In particular, no major differences were noted in the recommendations pertaining to the key aspects of the management of CAP: severity assessment, antibiotic choice and timeliness of treatment. Both guidelines recommend the use of:

A. clinical judgement in conjunction with the CURB-65 score (CRB65 score for primary care) to assess illness severity,

B. a single antibiotic as initial empirical therapy in patients with low severity CAP,

C. dual combination antibiotics comprising amoxicillin and a macrolide for patients with moderate severity CAP, and

D. dual combination antibiotics comprising a $\beta$-lactamase stable $\beta$-lactam (such as co-amoxiclav) and a macrolide for patients with high severity CAP.

In addition, both guidelines recommend that processes are be put in place to allow the radiological diagnosis and treatment of patients with CAP within $4 \mathrm{~h}$ of presentation to hospital.

\section{DISCUSSION}

The larger number of recommendations in the BTS Guideline compared with the NICE Pneumonia Guideline is partly due to differences in the scope of these two guidelines. Some of the areas covered in the BTS Guideline that were not evaluated for the NICE Guideline include the management of specific pathogens commonly associated with CAP, failure to improve and complications related to CAP.

Where the two guidelines overlap, there is considerable harmony. No major differences are 
Table 1 BTS Guideline recommendations that overlap with NICE Guideline recommendations where major differences exist

\section{BTS recommendation (number) [grade*] \\ (27) Investigations for legionella pneumonia are recommended for all patients with high severity CAP, for other patients with specific risk factors and for all patients with CAP during outbreaks. [D] \\ (28) Legionella urine antigen tests should be performed for all patients with high severity CAP. $[\mathrm{B}+]$}

(59) Review of patients in the community with CAP is recommended after $48 \mathrm{~h}$ or earlier, if clinically indicated. Disease severity assessment should form part of the clinical review. [D]

(60) Those who fail to improve after $48 \mathrm{~h}$ of treatment should be considered for hospital admission or chest radiography. [D]

(106) For patients managed in the community and for most patients admitted to hospital with low or moderate severity and uncomplicated pneumonia, 7 days of appropriate antibiotics is recommended. [C]
Related NICE recommendation (number)

(1.2.7) For patients with moderate- or high-severity community-acquired pneumonia:

take blood and sputum cultures and consider pneumococcal and legionella urinary antigen tests.

(1.2.13) Explain to patients with low-severity community-acquired pneumonia treated in the community, and, when appropriate, their families or carers, that they should seek further medical advice if their symptoms do not begin to improve within 3 days of starting the antibiotic, or earlier if their symptoms are worsening.

(1.2.23) Advise patients with community-acquired pneumonia to consult their healthcare professional if they feel that their condition is deteriorating or not improving as expected.

(1.2.10) Offer a 5-day course of a single antibiotic to patients with low-severity community-acquired pneumonia.

(1.2.15) Consider a 7- to 10-day course of antibiotic therapy for patients with moderate or high-severity community-acquired pneumonia.
Main difference

Patient groups for legionella testing

NICE recommends consideration of legionella urinary antigen tests for patients with both moderate and high severity CAP, not just high severity CAP.

NICE did not examine the value of legionella urinary antigen tests in patients with specific risk factors or in outbreak situations; the BTS recommendations in relation to these situations remain valid.

Timing of review

NICE recommendations are framed in the context of an outer limit (within 3 days), not a lower limit (after $48 \mathrm{~h}$ ).

\section{Duration of antibiotic therapy}

NICE recommends that in low severity CAP, a 5-day course of antibiotic therapy is offered, not a 7-day course.

NICE recommends that in moderate severity CAP,

a 7- to 10-day course of antibiotic therapy is considered, not just a 7 day course.

Both guidelines recommend a 7- to 10-day course of antibiotic therapy in high severity CAP.

${ }^{*} \mathrm{~A}$ full description of the guideline statement grades is provided in reference.

BTS, British Thoracic Society; CAP, community acquired pneumonia; NICE, National Institute for Health and Care Excellence.

evident in relation to the main pillars supporting the management of CAP. The NICE Guideline accordingly updates and strengthens the importance of these aspects of care. The BTS CAP Guideline remains valid with respect to these recommendations as does the BTS CAP Care Bundle, which contains these key elements. ${ }^{3}$

For the five BTS recommendations where major differences with the NICE Pneumonia Guideline exist, adherence to the NICE Pneumonia Guideline is recommended in preference to the BTS Guideline. With respect to the duration of antibiotic therapy for low severity CAP, in the UK many local hospital antibiotic guidelines currently already advocate a 5-day course as opposed to a 7-day course. This is in keeping with the broader objective of avoiding antibiotic overuse. ${ }^{4}$ The NICE Guideline adds valuable evidence-based support for this action.

The topic of non-invasive ventilation (NIV) or continuous positive airways pressure (CPAP) in patients with respiratory failure due to CAP was evaluated for the NICE Guideline and a NICE research recommendation was made but no practice recommendation. This stance from NICE is similar to the tone of the BTS Guidelines which recommends that 'Neither noninvasive ventilation (NIV) nor continuous positive airways pressure (CPAP) support is routinely indicated in the management of patients with respiratory failure due to CAP', and 'If a trial of non-invasive support is considered indicated in CAP, it must only be conducted in a critical care area where immediate expertise is available to enable a rapid transition to invasive ventilation'.

Many recommendations included in the BTS CAP Guideline covered topic areas that were not evaluated for the NICE Guideline. A review of the evidence base related to these recommendations is desirable in order to bring these recommendations up to date. In the meantime, these recommendations remain valid.
To help users clearly identify areas of overlap between the two guidelines, an annotated version of the BTS CAP Guideline has been produced and is available on the BTS website: http:// www.brit-thoracic.org.uk

\section{FUTURE DIRECTIONS}

The BTS Standards of Care Committee will, at a later date, decide on the timing and scope of a future update of the BTS CAP Guideline building on the excellence of the NICE Pneumonia Guideline. Topics to be considered for inclusion in future BTS CAP Guideline include the management of pneumonia associated with viral pathogens, specifically influenza, and aspiration pneumonia.

The relative lack of major differences between the NICE and BTS guidelines might be viewed positively as demonstrating coherence and consistency in guidelines. However, it also highlights the relative lack of substantial innovation and advancement over recent years in the evidence base related to the clinical management of CAP. More attention to translational and clinical research is merited if further improvements in the care of patients with CAP are to be gained. ${ }^{5}$

Correction notice This article has been corrected since it was published Online First. The provenance and peer review statement has been corrected.

Contributors WSL is the author with overall responsibility for the article. WSL, DLS, MPW and SAW all contributed to the development and drafting of the paper and all gave overall approval for submission.

\section{Competing interests None declared.}

Provenance and peer review Not commissioned; externally peer reviewed.

Open Access This is an Open Access article distributed in accordance with the Creative Commons Attribution Non Commercial (CC BY-NC 4.0) license, which permits others to distribute, remix, adapt, build upon this work non-commercially, and license their derivative works on different terms, provided the original work is 


\section{Chest clinic}

properly cited and the use is non-commercial. See: http://creativecommons.org/ licenses/by-nc/4.0/

\section{REFERENCES}

1 Lim WS, Baudouin SV, George RC, et al., Pneumonia Guidelines Committee of the BTS Standards of Care Committee. BTS guidelines for the management of community acquired pneumonia in adults: update 2009. Thorax 2009;64(Suppl 3):iii1-55.
2 NICE. National Institute for Health and Care Excellence. Pneumonia (including community acquired pneumonia). 2014. http://www.nice.org.uk/guidance/cg191

3 BTS Care Bundles for CAP and COPD. 2014. https://www.brit-thoracic.org.uk/auditand-quality-improvement/bts-care-bundles-for-cap-and-copd/

4 Department of Health. UK Five Year Antimicrobial Resistance Strategy 2013 to 2018. 2013.

5 Head MG, Fitchett JR, Newell ML, et al. Investment in pneumonia and pneumococcal research. Lancet Infect Dis 2014;14:1037-8. 